\title{
The relationship between acrolein and oxidative stress in COPD: in systemic plasma and in local lung tissue
}

This article was published in the following Dove Press journal: International Journal of Chronic Obstructive Pulmonary Disease

\author{
Masanori Yasuo' \\ Yunden Droma' \\ Yoshiaki Kitaguchi' \\ Michiko Ito' \\ Hitomi Imamura' \\ Masatomo Kawakubo ${ }^{2}$ \\ Masayuki Hanaoka' \\ 'The First Department of Internal \\ Medicine, Shinshu University School of \\ Medicine, Matsumoto, Nagano, Japan; \\ ${ }^{2}$ Department of Molecular Pathology, \\ Shinshu University Graduate School of \\ Medicine, Matsumoto, Nagano, Japan
}

Correspondence: Masanori Yasuo First Department of Internal Medicine, Shinshu University School of Medicine, 3-I-I Asahi, Matsumoto 390-862I, Japan Tel +8I 26337263 I

Fax +8I 263363722

Email yasumasa@shinshu-u.ac.jp
Purpose: Cigarette smoke produces a high level of acrolein, which is thought to be pathogenically involved in the development of chronic obstructive pulmonary disease (COPD). The present study investigated the pathological role of acrolein in the development of COPD.

Patients and methods: Acrolein concentration was measured in plasmas obtained from 47 patients with COPD and 18 current smokers without COPD, and in supernatants of homogenized lung tissues obtained from 10 never-smokers, 8 current smokers, and 8 patients with COPD by high-performance liquid chromatography. Oxidant status and antioxidant activity were measured using derivatives of reactive oxygen metabolite (d-ROM) and bio-antioxidant power (BAP), respectively, in the Free Radical Elective Evaluation FRAS4 system. In addition, immunohistochemistry was used to evaluate the over-presentation of acrolein in lung tissues of patients with COPD.

Results: Plasma concentrations of acrolein were significantly higher in the patients with COPD than the non-COPD smokers $(P<0.001)$, which significantly correlated with the oxidant status in patients with $\operatorname{COPD}(\mathrm{R}=0.69, P<0.05)$. Similar pathological alterations in acrolein concentrations were found in the lung tissue supernatants of patients with COPD, which significantly correlated with the oxidant status in patients with COPD. Furthermore, acrolein was strongly expressed in the lung tissues of patients with COPD.

Conclusion: The increased acrolein concentrations were highly involved in the pathogenesis of COPD through interference in the balance of oxidative stress versus antioxidant potentiality.

Keywords: acrolein, smoke, antioxidant potential, oxidative stress, plasma, lung tissue

\section{Introduction}

Chronic obstructive pulmonary disease (COPD) is a common, complex disorder associated with substantial morbidity and mortality that is characterized by progressive airflow obstruction due to varying degrees of bronchitis and emphysema. ${ }^{1}$ The pathogenesis of COPD results mainly from gene-environment interactions evoked by cigarette smoking through chronic inflammation of airways, proteolytic and anti-proteolytic imbalance, and oxidative stress. ${ }^{1}$ The production of oxidative stress results in critical consequences of catalytic events, such as epithelial permeability, lipid peroxidation, and cellular depletion of the antioxidant glutathione in alveoli, leading to apoptosis, injury, and inflammation in the pathogenesis of COPD. ${ }^{2}$ The antioxidants protect against the development of cigarette smoking 
extract (CSE)-induced emphysema in rats via the antioxidant pathway. ${ }^{3}$ In the clinical setting, targeting oxidative stress with antioxidants or boosting the endogenous levels of antioxidants is likely to be beneficial in the treatment of COPD. ${ }^{4}$

Cigarette smoking is the major etiological factor for COPD. ${ }^{1}$ Cigarette smoke is a complex mixture of more than 4700 known chemical compounds, including high concentrations of oxidants/free radicals and more than $10^{7}$ reactive organic compounds, such as high concentrations of acrolein and other carcinogenic organic compounds. ${ }^{5}$ The concentration of acrolein is estimated to be relatively high, approximately $80 \mu \mathrm{M}$, in the epithelial lining fluid (ELF) following complete inhalation of smoke from one cigarette in smokers. ${ }^{6}$ Moreover, the acrolein that endogenously produced from neutrophils in the process of inflammation and oxidative stress through myeloperoxidase activation makes the systemic level of acrolein higher than the estimated level from exogenous cigarette smoke. ${ }^{7}$ Pathogenically, acrolein is a highly reactive aldehyde, a nasal and tracheobronchial airway irritant with toxicity directly involved in the development of COPD. ${ }^{4,7}$ The acrolein concentrations in exhaled breath condensate and induced sputum have been demonstrated to be higher in patients with COPD than in healthy nonsmokers. ${ }^{8}$ In addition, elevated levels of acrolein have been found in the bronchoalveolar lavage (BAL) fluid of COPD patients. ${ }^{9}$ However, the levels of acrolein in human lung tissue and plasma from patients with COPD have not yet been reported; thus, the role of the acrolein in the pathogenesis of COPD is still uncertain.

In order to comprehensively understand the role of acrolein in the pathogenesis of COPD, we examined the plasma levels of acrolein and investigated the correlation between plasma acrolein levels and oxidative stress in patients with COPD. In addition, acrolein and oxidative stress were measured in lung tissue supernatants from another group of patients with COPD and the correlations were analyzed.

\section{Materials and methods}

This study was approved by the Ethics Committee of Shinshu University (Permission number 300, Date of approval 8 May 2013). The study protocols were performed in accordance with the principles outlined in the Declaration of Helsinki of the World Medical Association. Written informed consent was obtained from all patients.

\section{Patients}

The patients with COPD were recruited from the Respiratory Center of Shinshu University Hospital (Matsumoto, Japan). The diagnosis of COPD was based on smoking history, symptoms, including dyspnea on exertion, and pulmonary function characterized by persistent airflow obstruction, which was in accordance with the Global Initiative for Chronic Obstructive Lung Disease (GOLD) guidelines. ${ }^{10}$

The control subjects were smokers without COPD (non-COPD smokers). They were recruited from a Japanese population undergoing regular health check-ups at Misayama Hospital and Shinmachi Hospital in Japan. The spirometry measurements were performed to exclude COPD in these control subjects.

All COPD patients and controls with the following disorders were excluded: late sequelae of pulmonary tuberculosis, diffuse panbronchiolitis, sinobronchitis, bronchiectasis, bronchiolitis obliterans due to autoimmune disease, and asthma.

\section{Diagnosis and classification of COPD}

Spirometry and carbon monoxide diffusion capacity (DLco) were measured by a pulmonary function test (Chest-55 V, Chest Co. Ltd., Tokyo, Japan). Spirometry values were expressed as a percentage of the predicted values calculated by the formula for the Japanese population. ${ }^{11}$ Airflow limitation was defined as a ratio of forced expiratory volume in one second $\left(\mathrm{FEV}_{1}\right)$ to forced vital capacity (FVC) of less than $70 \%\left(\mathrm{FEV}_{1} / \mathrm{FVC}<70 \%\right)$ after inhalation of short-acting $\beta 2$-agonists. ${ }^{1,10}$ The severity of airflow limitation was determined according to the GOLD classifications: all stages had $\mathrm{FEV}_{1} / \mathrm{FVC}<70 \%$, and stage I had $\% \mathrm{FEV}_{1} \geq 80 \%$, stage II $50 \% \leq \%$ $\mathrm{FEV}_{1}<80 \%$, stage III $30 \% \leq \% \mathrm{FEV} 1<50 \%$, and stage IV $\%$ FEV $1<30 \%$. ${ }^{1,10}$ Other pulmonary function parameters, such as peak expiratory flow rate (PEFR), functional residual capacity (FRC), residual volume (RV), total lung capacity (TLC), and vital capacity (VC), were also measured.

\section{Plasma samples}

Venous blood samples were obtained from all patients and controls and collected into commercially available anticoagulant-treated tubes. The plasma was separated from the whole blood by centrifugation for 10 mins at 3,000 rpm using a refrigerated centrifuge. The plasma samples were 
stored in a freezer at $<-80{ }^{\circ} \mathrm{C}$ until the measurement of acrolein, derivatives of reactive oxygen metabolite (d-ROM), and bio-antioxidant power (BAP).

\section{Human lung tissue samples}

In order to investigate the acrolein status in the lung tissues of COPD patients, specimens were obtained from another group of patients with COPD combined with lung cancer. The COPD in this group was diagnosed by smoking history, lung function test, and high-resolution computed tomography (CT) before thoracic surgical treatment for lung cancer. Written informed consent was obtained from each of the patients. The lung tissues were sampled from the resected lung lobes at a site distant from the cancerous lesions and the margin of the lung specimen histologically confirmed to be in absence of cancerous tissue. Metastasis and inflammation were excluded as well. Clinically, the patients in this group were in COPD stage I and II based on the GOLD guidelines. ${ }^{1,10}$

One part of the lung specimen was prepared in a paraffin-embedded block for immunohistochemistry. The remaining part was homogenized and the supernatant was separated after two cycles of centrifugation at $10,000 \mathrm{~g}$ for $10 \mathrm{~min}$. The lung tissue supernatant was stored in a freezer at $<-80{ }^{\circ} \mathrm{C}$ until the measurement of acrolein, d-ROM, and BAP.

\section{Measurement of acrolein}

Preparation of the dinitrophenylhydrazone derivatives Acrolein (Sigma-Aldrich Japan, Tokyo, Japan) was derivatized with DNPH, a procedure as described previously by Andreoli et al. ${ }^{12}$ Briefly, DNPH $(10 \mathrm{mg}$ ) was dissolved in $4 \mathrm{~mL}$ of acetonitrile and acidified with $80 \mu \mathrm{L}$ of formic acid. The DNPH solution ( $12 \mathrm{mM})$ was stored at $4{ }^{\circ} \mathrm{C}$ until use. Derivatization of aldehydes was performed by mixing $100 \mu \mathrm{L}$ of the standard solutions (or samples) with $100 \mu \mathrm{L}$ of the derivatizing agent and incubating at room temperature for $1 \mathrm{~h}$. Then, $20 \mu \mathrm{L}$ of derivatized samples were directly injected onto the LC/MS system.

\section{Liquid chromatography/mass spectrometry analysis} Shimadzu LC/MS consisted of Shimadzu SPD-M10Avp PDA Detector, Shimadzu LC-10ADvp solvent delivery system, CTO-10ACvp clumn oven, Shimadzu LCMS-2010A MS Detector, and Shimadzu LCMSsolution software (Tokyo, Japan). Chromatography of DNPH derivatives was performed on a supelcosil LC-18-DB HPLC column (SigmaAldrich, Milan, Italy) using $20 \mathrm{mM}$ aqueous acetic acid and methanol as mobile phase under gradient elution conditions at a flow rate of $0.80 \mathrm{~mL} / \mathrm{min}$. Gradient program: from $43 \%$ to $98 \%$ methanol in $3.2 \mathrm{~min}$, linear gradient, and then hold for $2.5 \mathrm{~min}$. The analytes were ionized by APCI in negativeion mode for acrolein. APCI production spectra were obtained by flow injections of standards of the DNPH derivatives (about $50 \mu \mathrm{M}$ ) dissolved in a 1:1 (v/v) acetonitrile/aqueous $\mathrm{HCOOH}(2 \%)$ mixture and acquired in the scan range $\mathrm{m} / \mathrm{z}$ 70-350. Detection for sample analyses was obtained in selected-ion monitoring (SIM) mode m/z 235.

\section{Assessment of oxidant status and antioxidant activity}

The oxidant status and antioxidant activity were measured in plasma and lung tissue supernatant by a d-ROM test and BAP test, respectively, using the Free Radical Elective Evaluation FRAS4 system (Diacron International, Grosseto, Italy) according to the manufacturer's instructions. The d-ROM test mainly measures hydroperoxides, products of dehydrogenation and peroxidation of several cellular components. ${ }^{13}$ The d-ROM levels indicated the oxidant status, which was expressed in conventional Carratelli units (U Carr), with $1 \mathrm{U}$ Carr corresponding to $0.8 \mathrm{mg} / \mathrm{L}$ of $\mathrm{H}_{2} \mathrm{O}_{2}$. The BAP test provides the biological antioxidant potential, with measurement based on the ability of reduction by anti-oxidant substances, including glutathione. ${ }^{14}$ The BAP level was expressed as $\mu \mathrm{mol} / \mathrm{L}$.

\section{Immunohistochemistry of protein-bound acrolein in lung tissues}

Since acrolein rapidly reacts with protein such as histidine residues of carbonic anhydrase to bound with protein, we can detect the protein-bound acrolein by means of immunohistochemistry. Uchida et al verified the presence of protein-bound acrolein in vivo, and produced mAb5F6 antibody that was against the acrolein-modified keyhole limpet hemocyanin, ${ }^{15}$ in which the acrolein-lysine adduct, $\mathrm{N} \varepsilon$-(3-formyl-3,4-dehydropiperidino) lysine (FDP-lysine) constituted an epitope of the antibody. ${ }^{15}$ In the present study, the antibody (mAb5F6) was purchased from Japan Institute for the Control of Aging (JaICA: NIKKEN SEIL Co. Ltd., Fukuroi, Japan) and the immunohistochemistry was performed according to the manufacturer's instruction. Briefly, the lung tissue sections were deparaffinized and then incubated in $1 \%$ hydrogen peroxide in methanol for $30 \mathrm{~min}$ at room temperature to block endogenous peroxidase activity. The sections were incubated overnight 
in Tris-buffered saline (TBS) containing mouse antibody for the FDP-lysine at a 1:1000 dilution. After washing with TBS, the sections were incubated with the biotinconjugated anti-mouse antibody (1:500; Dako, Glostrup, Denmark) for $1 \mathrm{hr}$, washed with TBS, and reacted with peroxidase-labeled avidin-biotin complex (Dako) for $30 \mathrm{~min}$. The reaction was visualized with the aid of a solution containing 0.01\% 3,3'-diaminobenzidine (Wako Pure Chemical, Osaka, Japan) and $0.005 \%$ hydrogen peroxide. Counterstaining was carried out with hematoxylin.

\section{Statistical analysis}

All data are expressed as mean \pm standard error (SE). Data were evaluated by ANOVA using statistical software (version 14.0, SPSS Inc., Chicago, IL, USA). Correlations were analyzed by the Pearson correlation coefficient. Significance was set at $P<0.05$ in a two-tailed test.

\section{Results}

\section{Plasma acrolein, d-ROM, and BAP levels}

A total of 47 patients with COPD were recruited for this study, including 12 patients considered stage I, 12 patients stage II, 11 patients stage III, and 12 patients stage IV. The controls were 18 non-COPD smokers. Average age and the gender ratio did not differ significantly between the COPD group and non-COPD smokers (Table 1). There were 36 former smokers and 11 current smokers in the COPD group and 9 former smokers and 9 current smokers in the non-COPD smoker group (Table 1). The smoking history was significantly heavier in the patients with COPD than the non-COPD smokers $(P<0.001)$, and $\mathrm{FEV}_{1}$ and $\mathrm{FEV}_{1} / \mathrm{FVC}$ were significantly different between the groups (Table 1).

The plasma acrolein concentration was significantly higher in the patients with COPD than the non-COPD smokers $(P<0.001$, Figure 1A) and in stage I and IV COPD than non-COPD smokers $(P<0.01$, Figure $1 \mathrm{~B})$. However, the patients with stage II and III COPD did not have significantly different plasma acrolein concentrations than the non-COPD smokers (Table 1, Figure 1B), probably due to the small sample size and large range in $\mathrm{SE}$ in these two groups.

The plasma d-ROM level was significantly higher in the patients with COPD than the non-COPD smokers $(P<0.01$, Table 1). However, no significant differences were found in the plasma d-ROM levels between the COPD stage subgroups and non-COPD smokers (Table 1). Plasma BAP levels did not significantly differ between the COPD groups and non-COPD smokers (Table 1).

\section{Correlation of acrolein levels with oxidant status in plasma}

The plasma acrolein levels significantly positively correlated with the d-ROM levels in the stage III and IV COPD subgroup ( $\mathrm{R}=0.69, P<0.05$, Figure 2C). However, the plasma acrolein levels did not significantly correlate with the plasma d-ROM levels in the stage I and II COPD subgroup ( $\mathrm{R}=0.04, P>0.05$, Figure $2 \mathrm{~B}$ ). The

Table I Characteristics, spirometry data, and measurements of acrolein and oxidation for the subjects in the plasma study

\begin{tabular}{|c|c|c|c|c|c|c|}
\hline & Stage I & Stage II & Stage III & Stage IV & $\begin{array}{l}\text { Total COPD } \\
\text { (Stage I-IV) }\end{array}$ & $\begin{array}{l}\text { Non-COPD } \\
\text { smokers }\end{array}$ \\
\hline Number (n) & 12 & 12 & 11 & 12 & 47 & 18 \\
\hline Male/Female (n) & $12 / 0$ & $12 / 0$ & $11 / 0$ & $12 / 0$ & $47 / 0$ & $16 / 2$ \\
\hline Age (years) & $74.7 \pm 1.7 *$ & $70.1 \pm 1.6$ & $72.5 \pm 1.7$ & $70.3 \pm 1.2$ & $71.9 \pm 0.8$ & $68.4 \pm 1.5$ \\
\hline FS/CS (n) & $10 / 2$ & $7 / 5$ & $8 / 3$ & $\mathrm{II} / \mathrm{I}$ & $36 / 11$ & $9 / 9$ \\
\hline $\begin{array}{l}\text { Smoking history- } \\
{ }^{\dagger} \text { (pack-years) }\end{array}$ & $60.1 \pm 8.4$ & $49.4 \pm 4.0$ & $62.3 \pm 8.0$ & $82.5 \pm 9.9 * * *$ & $63.6 \pm 4.2 * * *$ & $45.9 \pm 2.0$ \\
\hline FEV , (\% pred) & $92.0 \pm 3.5$ & $67.0 \pm 2.0 * * *$ & $37.6 \pm 3.8^{* * *}$ & $22.9 \pm 1.4^{* * *}$ & $56.5 \pm 1.7 * * *$ & $83.9 \pm 2.3$ \\
\hline FEV $/$ FVC (\%) & $62.1 \pm 1.1 * * *$ & $58.3 \pm 1.1 * * *$ & $44.3 \pm 1.9 * * *$ & $34.9 \pm 5.9 * * *$ & $49.9 \pm 1.9 * * *$ & $77.2 \pm 1.2$ \\
\hline Acrolein (ng/ml) & $135.3 \pm 19.2 * *$ & $126.7 \pm 27.7$ & $138.5 \pm 53.8$ & $288.9 \pm 67.4^{* *}$ & $217.9 \pm 41.2^{* * *}$ & $67.9 \pm 11.2$ \\
\hline d-ROM (Unit Carr) & $117.00 \pm 9.13$ & $105.64 \pm 12.37$ & II $5.44 \pm 13.80$ & $127.00 \pm 18.13$ & $114.74 \pm 6.37 * *$ & $92.8 \mathrm{I} \pm 5.0 \mathrm{I}$ \\
\hline BAP ( $\mu \mathrm{mol} / \mathrm{L})$ & $5517.72 \pm 141.26$ & $5250.14 \pm 133.89$ & $5264.5 I \pm 170.77$ & $54 \mid 3.93 \pm 258.03$ & $5350.72 \pm 81.78$ & $5548.64 \pm 103.35$ \\
\hline
\end{tabular}

Notes: Data are expressed as mean \pm SE. Stage I IV indicate the severity of the airflow limitation according to spirometric (GOLD) classifications as follows: all stages had $\mathrm{FEV}_{1} / \mathrm{FVC}<70 \%$; in addition, stage I: $\% \mathrm{FEV}_{1} \geq 80 \%$; stage II: $50 \% \leq \% \mathrm{FEV},<80 \%$; stage III: $30 \% \leq \% \mathrm{FEV},<50 \%$; and stage IV: $\% \mathrm{FEV},<30 \%$. $* P<0.05$ vs non-COPD smokers, $* * P<0.01$ vs non-COPD smokers, $* * * P<0.001$ vs non-COPD smokers based on ANOVA. $\dagger 10$ pack-year is equal to smoking 20 cigarettes ( 1 pack) per day for 10 year. Abbreviations: $\mathrm{FEV}_{1}$, forced expiratory volume in I second; FVC, forced vital capacity; d-ROM, reactive oxygen metabolite; BAP, bioantioxidant power; FS, former smoker; $\mathrm{CS}$, current smoker; $\mathrm{n}$, number of subjects. 
A

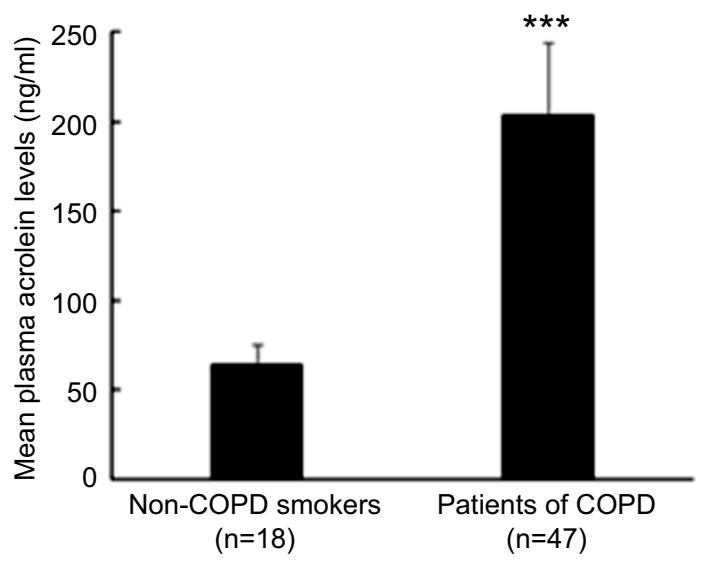

B

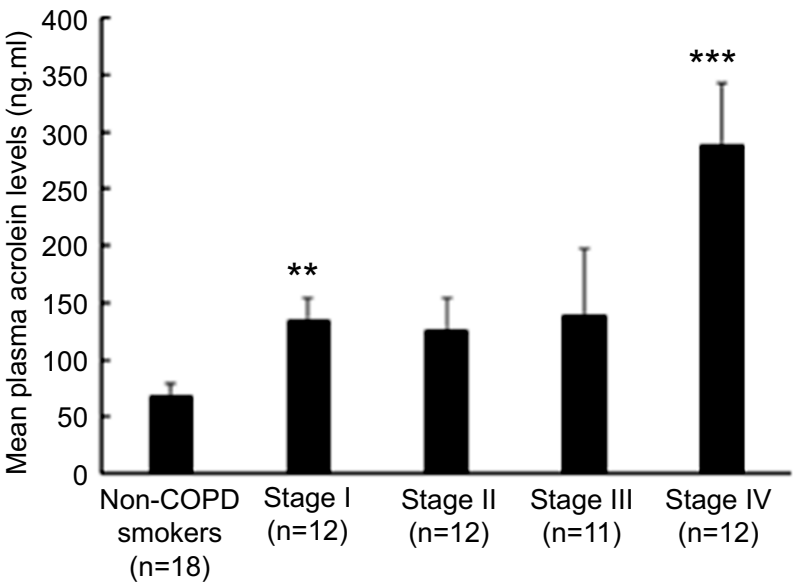

Figure I Plasma acrolein concentrations in non-COPD smokers and patients with COPD. (A) Non-COPD smokers and total patients with COPD. (B) Non-COPD smokers and stage I, II, III, and IV COPD by the GOLD classification. $* * P<0.0$ I vs non-COPD smokers; $* * * P<0.00 \mathrm{I}$ vs non-COPD smokers. The bars indicate standard error (SE).

A

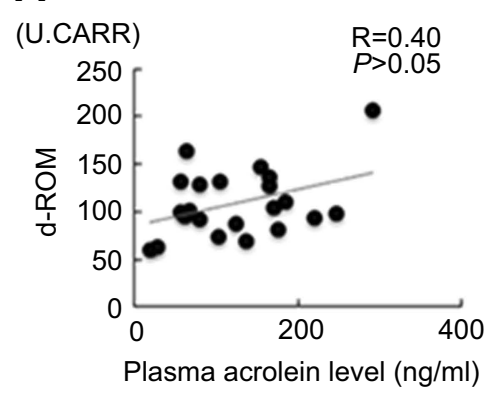

D

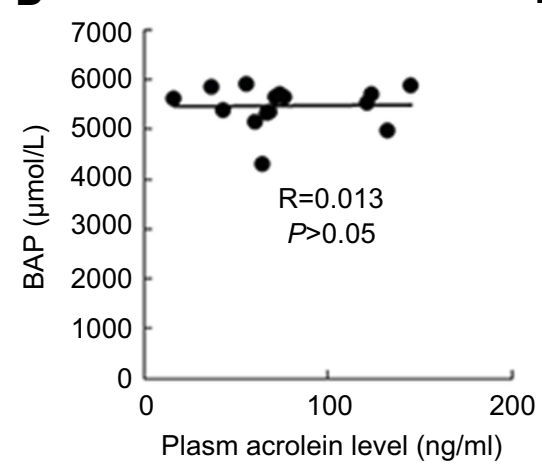

B

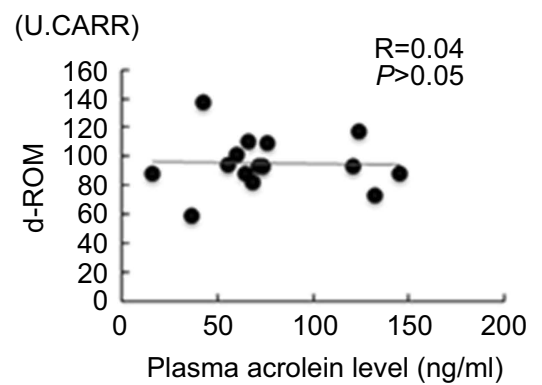

E

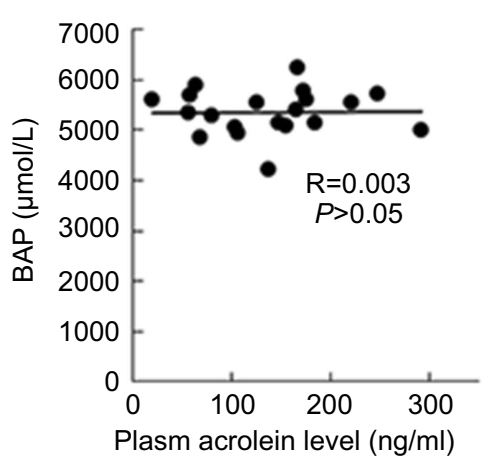

C

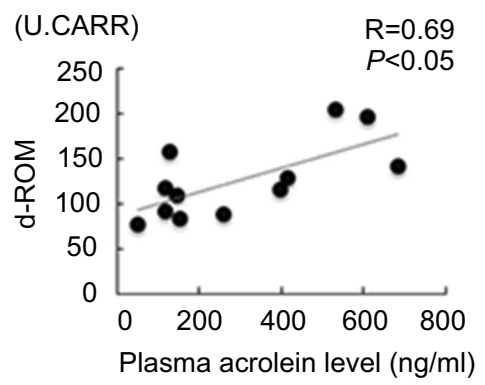

$\mathbf{F}$

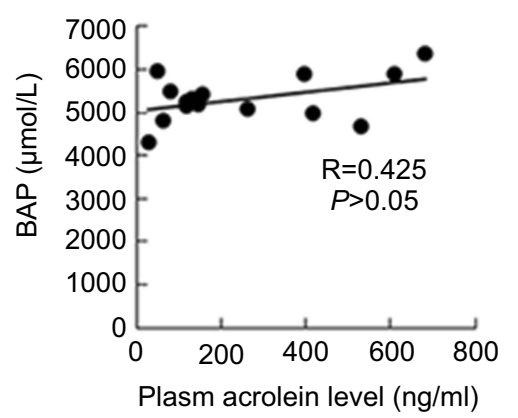

Figure 2 Correlation of acrolein concentrations with the derivatives of reactive oxygen metabolite (d-ROM) and bio-antioxidant power (BAP) levels in plasma. (A) MonCOPD smokers $(R=0.40, P>0.05)$. (B) Stage I and II COPD $(R=0.04, P>0.05)$. (C) Stage III and IV COPD $(R=0.69, P<0.05)$. (D) Non-COPD smokers $(R=0.013, P>0.05)$. (E) Stage I and II COPD $(R=0.003, P>0.05)$. (F) Stage III and IV COPD $(R=0.425, P>0.05)$.

non-COPD smokers did not show such a significant correlation between the plasma acrolein concentration and $\mathrm{d}$ ROM levels ( $\mathrm{R}=0.40, P>0.05$, Figure $2 \mathrm{~A}$ ). In addition, we did not detect any correlations between smoking history and d-ROM in the COPD group (total COPD,
$\mathrm{R}=0.15$; Stage $\mathrm{I}$ and II, $\mathrm{R}=0.17$; and Stage III and IV, $\mathrm{R}=0.07$ ).

In addition, the plasma acrolein concentrations did not show any significant correlations with the anti-oxidative potential of BAP in non-COPD smokers, moderate COPD 
(stage I and II), and severe COPD (stage III and IV, Figure 2D-F, $P>0.05$ in all groups).

\section{Correlation of plasma acrolein}

\section{concentrations with smoking history}

The plasma acrolein concentrations significantly positively correlated with smoking history in the stage III and IV COPD subgroup ( $\mathrm{R}=0.46, P<0.05$, Figure $3 \mathrm{~B}$ ), but did not significantly correlate with smoking history in the stage I and II COPD subgroup ( $\mathrm{R}=0.13, P>0.05$, Figure $3 \mathrm{~A}$ ).

\section{Correlation of plasma acrolein levels with pulmonary function}

The plasma acrolein levels significantly positively correlated with FRC (Figure 4A, R $=0.47, P<0.05$ ) and RV (Figure $4 \mathrm{~B}, \mathrm{R}=0.40, P<0.05$ ) in the stage III and IV COPD subgroup. However, no significant correlation was found between the plasma acrolein levels and lung function based on FRC and RV in the stage I and II COPD subgroup.

\section{Acrolein, d-ROM, and BAP levels in human lung tissue supernatants}

Lung tissue specimens were obtained from another group of 26 patients with COPD and lung cancer treated by thoracic surgery. The patients were divided into three groups based on smoking history, lung function test, and high-resolution CT: non-COPD never-smokers $(\mathrm{n}=10)$, non-COPD smokers $(n=8)$, and COPD patients $(n=8)$. Data on demographics, lung function test, acrolein concentration, and d-ROM and BAP levels in the lung tissue supernatants are given in

A

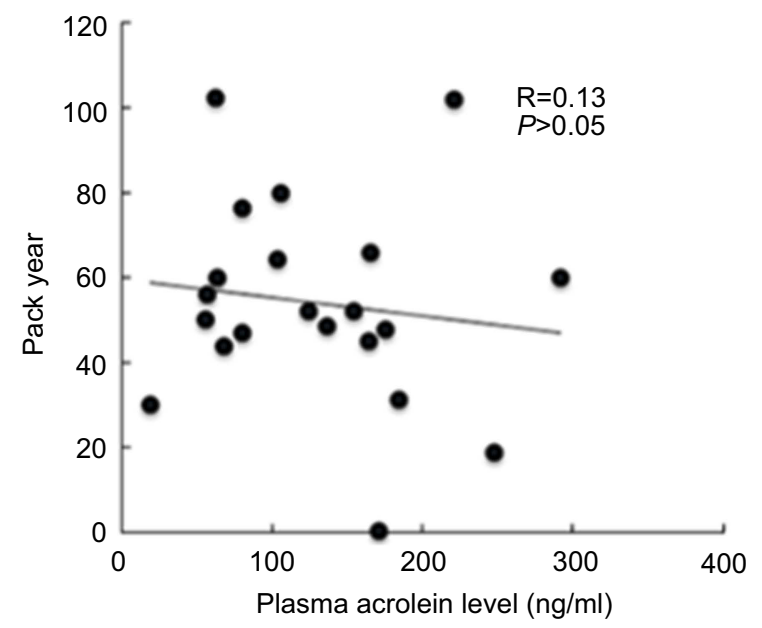

Table 2. The average age was similar for the three groups $(P>0.05)$. However, significantly more males were present among the non-COPD smokers and COPD group than the non-COPD never-smokers ( $P=0.00019)$. Because $9 / 10$ of the non-COPD never-smokers were female patients with lung cancer of adenocarcinoma. The non-COPD smokers and COPD patients were heavy smokers. There were 6 former smokers and 2 current smokers in the non-COPD smoker group and 4 former smokers and 4 current smokers in the COPD group (Table 2). There were no significant differences of acrolein concentration between the former and current smokers $(P=0.77$ in the non-COPD smoker group, and $P=0.85$ in the COPD group). Spirometry measurements indicated that the patients in the COPD group were classified as stage I and II according to the GOLD guidelines (Table 2). ${ }^{1,10}$

The acrolein concentrations in lung tissue supernatants were significantly higher in the COPD group $(P=0.003)$ and non-COPD smokers $(P=0.04)$ than the never-smokers (Table 2 and Figure 5). The d-ROM levels in lung tissue supernatants were significantly higher in the COPD group $(P=0.004)$ and non-COPD smokers $(P=0.03)$ than the neversmokers. However, no significant differences were found in the lung BAP levels between these three groups (Table 2).

\section{Correlation of acrolein levels with oxidant status in human lung tissue supernatants}

The acrolein concentrations in lung tissue supernatants significantly positively correlated with the d-ROM levels in never-smokers (Figure 6A, $\mathrm{R}=0.69, P<0.05$ ), nonCOPD smokers (Figure 6B, $\mathrm{R}=0.93, P<0.05$ ), and the

B

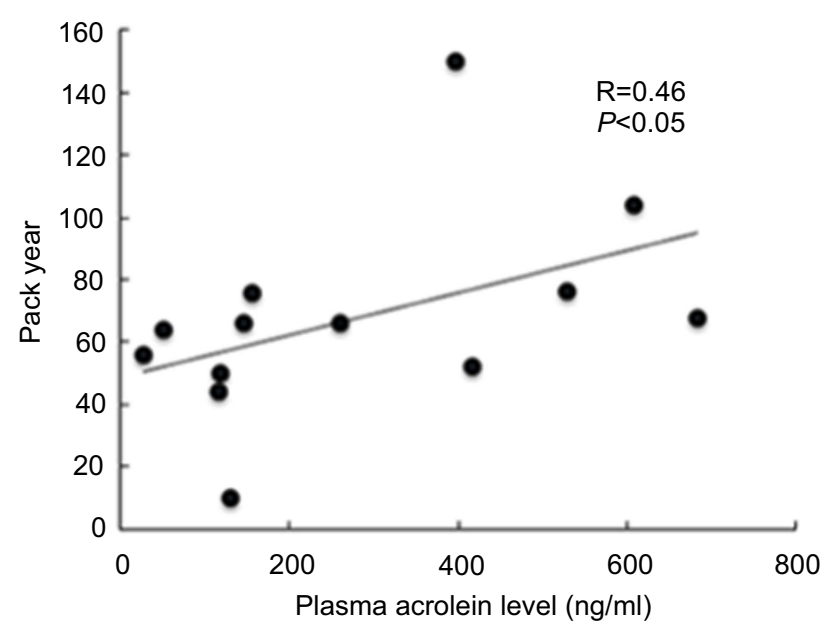

Figure 3 Correlation of plasma acrolein levels with smoking history. (A) Stage I and II COPD $(R=0.13, P>0.05)$. (B) Stage III and IV COPD ( $=0.46, P<0.05)$. 
A

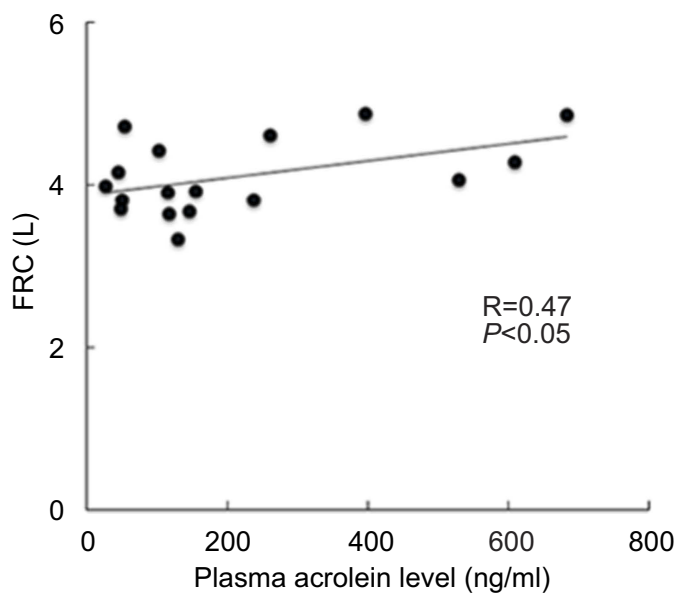

B

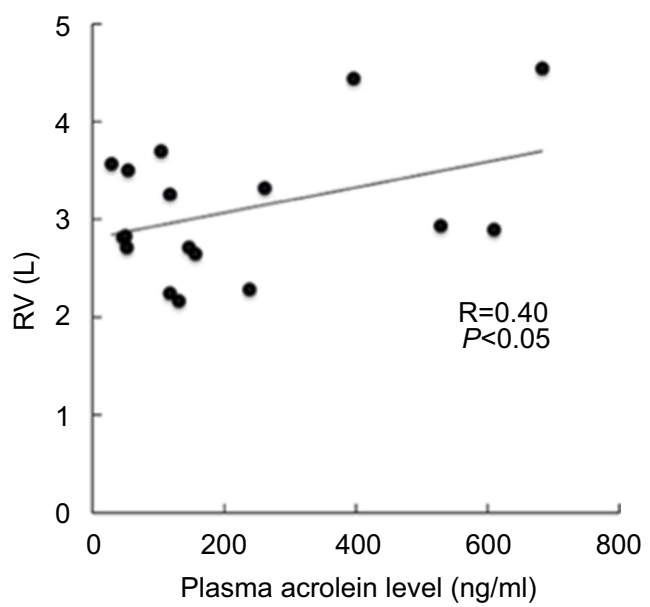

Figure 4 Corrected plasma acrolein levels with pulmonary functions in stage III and stage IV COPD. (A) Functional residual capacity $(F R C)(R=0.47, P<0.05)$. (B) Residual volume $(R V)(R=0.40, P<0.05)$.

Table 2 Characteristics, spirometry data, and measurements of acrolein and oxidation for the subjects in the lung study

\begin{tabular}{|l|l|l|l|}
\hline & Non-COPD smokers & COPD & Non-COPD Never smokers \\
\hline Number & 8 & 8 & 10 \\
Male/Female & $6 / 2 * * *$ & $8 / 0$ & $1 / 9$ \\
Age (years) & $67.3 \pm 4.1$ & $70.1 \pm 2.6$ & $69.1 \pm 3.8$ \\
FS/CS (n) & $6 / 2$ & $4 / 4$ & NA \\
Smoking history (pack-years) & $35.3 \pm 6.1 * * * *$ & $68.4 \pm 16.9 * * *$ & 0 \\
FEV (\% pred) & $101.2 \pm 6.4$ & $71.3 \pm 6.8^{* * * *}$ & $107.9 \pm 5.5$ \\
FEV /FVC (\%) & $76.4 \pm 2.6$ & $61.1 \pm 2.7 * * * *$ & $78.7 \pm 0.9$ \\
Acrolein (ng/ml) & $35.58 \pm 11.30^{*}$ & $32.53 \pm 5.59 * * *$ & $8.11 \pm 2.04$ \\
d-ROM (Unit Carr) & $45.38 \pm 1.52^{*}$ & $48.88 \pm 1.94 * * *$ & $41.10 \pm 0.85$ \\
BAP ( $\mu$ mol/L) & $3652.36 \pm 34.28$ & $3568.94 \pm 104.79$ & $3505.59 \pm 39.77$ \\
\hline
\end{tabular}

Notes: Data are expressed as mean \pm SE. $* P<0.05$ vs non-COPD never-smokers, $* * * P<0.005$ vs non-COPD never- smokers, and $* * * * P<0.001$ vs non-COPD never-smokers based on ANOVA. $\dagger 10$ pack-year is equal to smoking 20 cigarettes (I pack) per day for 10 year.

Abbreviations: $\mathrm{FEV}_{1}$, forced expiratory volume in I second; FVC, forced vital capacity; d-ROM, reactive oxygen metabolite; BAP, bioantioxidant power; FS, former smoker; $\mathrm{CS}$, current smoker; $\mathrm{n}$, number of subjects; NA, not associated.

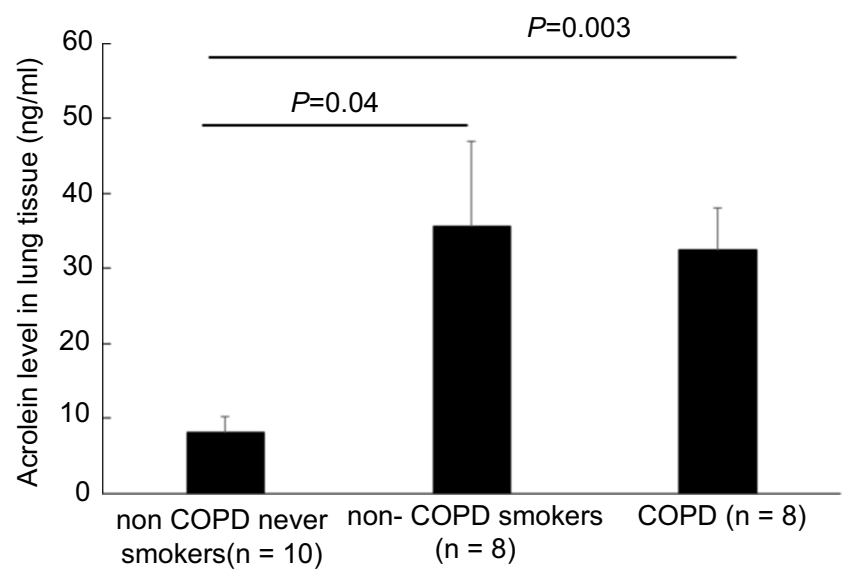

Figure 5 Acrolein concentrations in human lung tissues in non-COPD never-smokers, non-COPD smokers, and patients with COPD. P-values are based on comparisons to the non-COPD never smokers. The bars indicate standard error (SE).
COPD group (Figure $6 \mathrm{C}, \mathrm{R}=0.59, P<0.05$ ). However, the acrolein levels significantly negatively correlated with the BAP levels in the non-COPD smokers (Figure 6E, $\mathrm{R}=-0.64, P<0.05)$. The acrolein levels exhibited no correlations with BAP levels in the never-smokers (Figure $6 \mathrm{D}, \mathrm{R}=0.19, P<0.05$ ) and the COPD group (Figure $6 \mathrm{~F}, \mathrm{R}=0.38, P>0.05$ ).

\section{Correlation of acrolein levels in lung tissue supernatants with the peak expiratory flow rate (PEFR) in COPD}

Among the lung function parameters measured in the present study, the PEFR significantly negatively correlated with acrolein concentration in lung tissue supernatants 

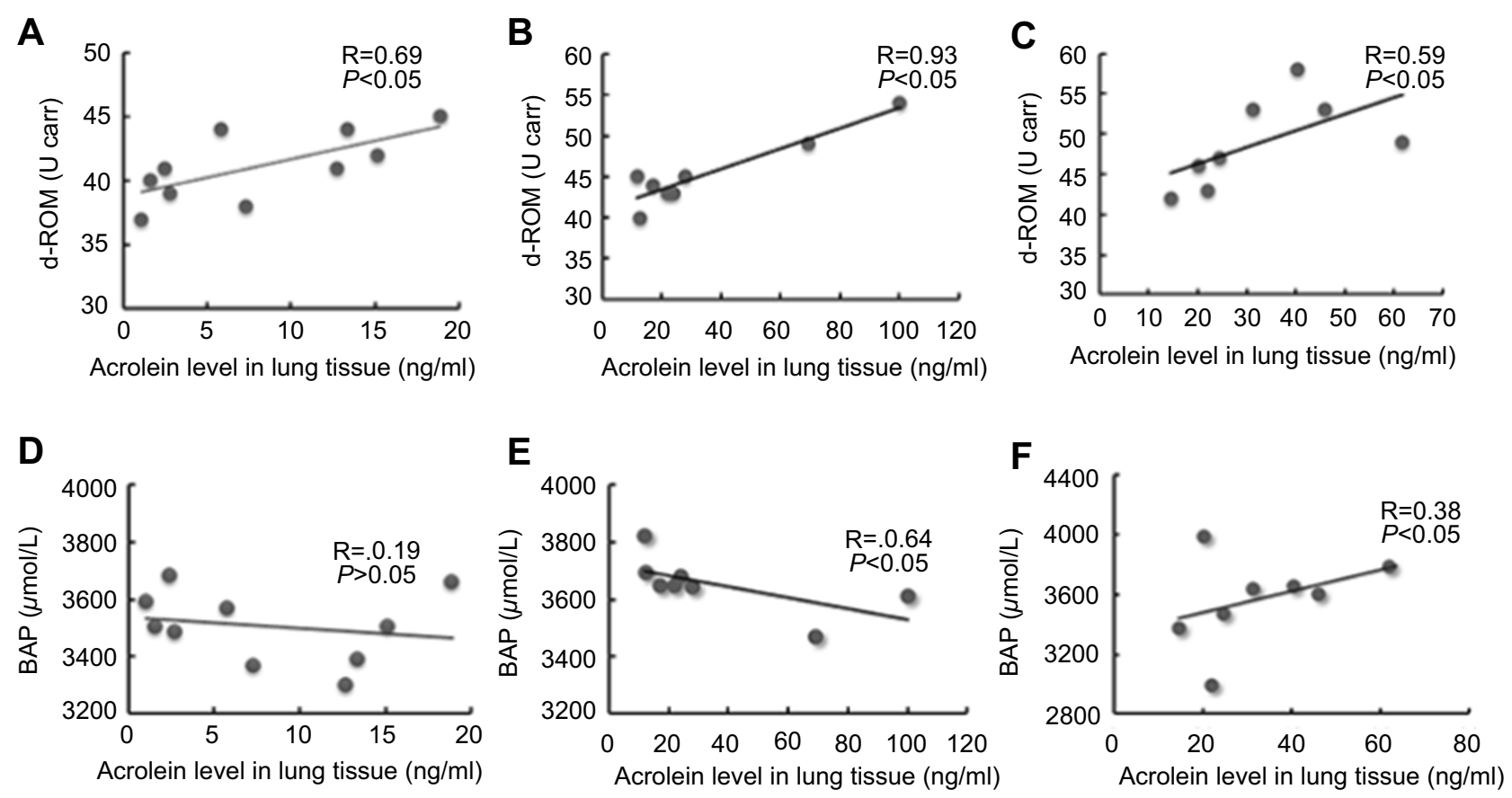

Figure 6 Correlation of acrolein concentration with the derivatives of reactive oxygen metabolite (d-ROM) and bio-antioxidant power (BAP) in human lung tissues. (A) Non-COPD never-smokers $(R=0.69, P<0.05)$. (B) Non-COPD smokers $(R=0.93, P<0.05)$. (C) Patients with COPD $(R=0.59, P<0.05)$. (D) Non-COPD never smokers $(R=$ $-0.19, P>0.05)$. (E) Non-COPD smokers $(R=-0.64, P<0.05)$. (F) Patients with COPD $(R=0.38, P<0.05)$.

from stage I and II COPD patients $(\mathrm{R}=-0.62, P<0.05$, Figure 7).

\section{Immunohistochemistry}

In pathology, immunohistochemistry showed that acrolein was expressed in the lung tissues of both the COPD group (Figure 8C) and non-COPD smokers (Figure 8B) but few in the lung tissues of never smokers (Figure 8A).

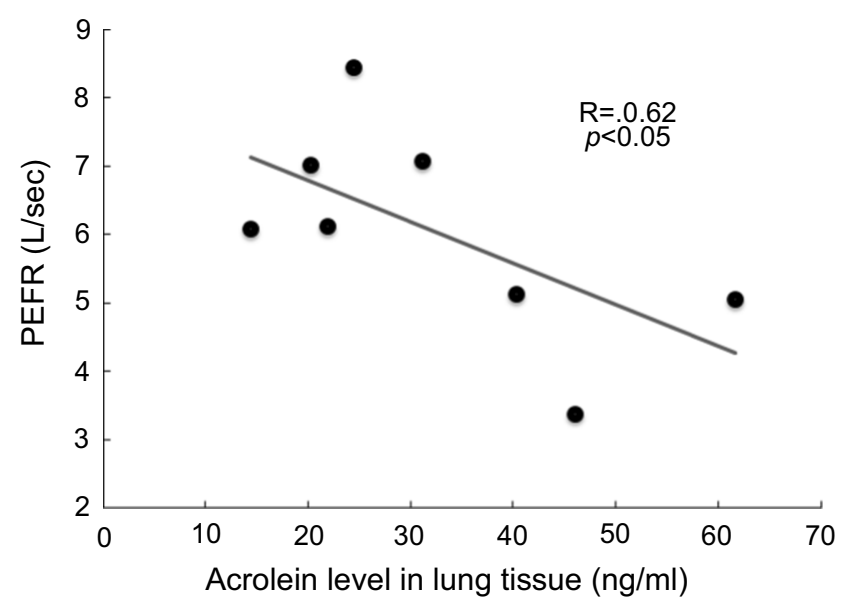

Figure 7 Corrected acrolein concentrations in lung tissues with respiratory expiratory flow rate $(P E F R)$ in the stage I and stage II COPD $(R=-0.62, P<0.05)$.

\section{Discussion}

Given the relative abundance of acrolein in cigarette smoke and its toxic potential to induce oxidant stress, ${ }^{7}$ it is surprising how little is known about the acrolein levels in plasma and lung tissues and their correlations with oxidant stress in patients with COPD. The remarkable findings of the present study were that plasma acrolein levels significantly increased in patients with COPD, and that this increase significantly positively correlated with smoking history, increased FRC and RV, and the oxidant stress status in patients with severe COPD. These results suggest that a longer smoking history resulted in higher plasma acrolein levels, which then induced oxidative stress, resulting in small airway obstruction $\left(\mathrm{FEV}_{1} / \mathrm{FVC}\right.$ $<70 \%$ ) and hyperinflation of lung functions in the pathogenesis of COPD.

The plasma acrolein levels are altered by both exogenous sources (eg, incomplete combustion of petrol, wood, and plastic, frying of foods in oils) and endogenous sources (eg, lipid peroxidation and polyamine metabolism). ${ }^{7}$ Acrolein is soluble in water, alcohol, and diethyl ether, and can travel across membranes by passive diffusion. Exogenous acrolein from a tobacco source easily passes the alveolar membrane into the blood circulation, ${ }^{7}$ inducing neutrophil activation and producing 


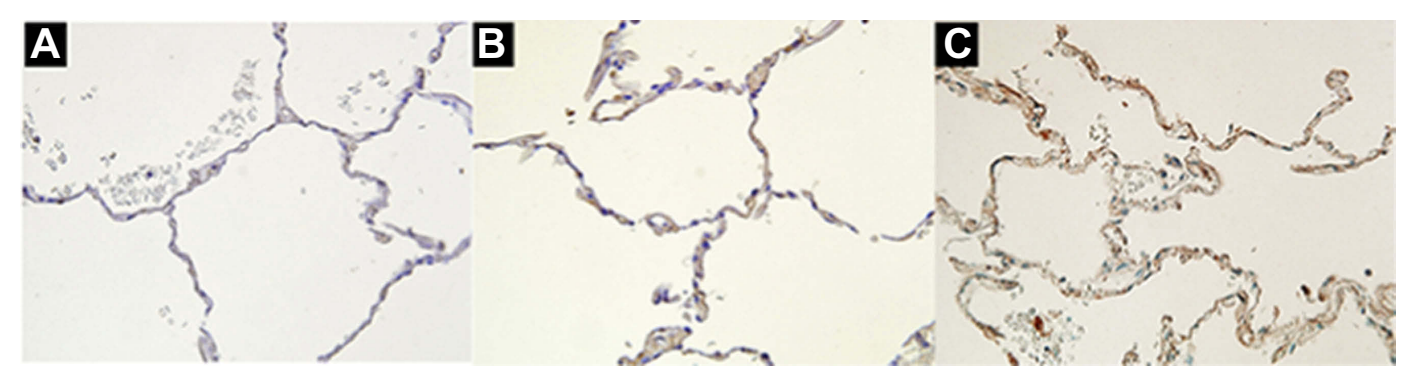

Figure 8 Immunohistochemistry of acrolein in human lung tissues. (A) Very weak staining in lung tissue from non-COPD never smokers. (B) Moderate staining in lung tissue from non-COPD smokers. (C) Moderate staining in lung tissue from patients with stage I and stage II COPD. Original magnification $x 400$.

endogenous acrolein via myeloperoxidase and polyamine metabolism. $^{7}$ The present evidence of increased acrolein (exogenous and endogenous) in systemic blood circulation supports the proposal of COPD as a systemic disease. ${ }^{16,17}$ This is clinically significant for patients, as the extrapulmonary manifestations of COPD are common and/or may have significant implications for the prognosis, and systematic screening and appropriate management can be provided for optimal medical care. ${ }^{17}$

The lungs are affected directly by the toxicity of acrolein produced by smoking tobacco. The main urinary metabolite of acrolein, 3-hydroxypropyl mercapturic acid, is found at about twice the level in the urine of smokers compared to non-smokers. ${ }^{18}$ Acrolein (namely 2-propenal), is highly irritating to respiratory passages. ${ }^{7}$ Because of its solubility and reactivity with respiratory-lining fluid and cellular macromolecules, acrolein alters gene regulation, inflammation, mucociliary transport, and alveolar-capillary barrier integrity in the respiratory system- ${ }^{7}$ Chronic exposure to low concentrations of acrolein results in bronchial hyperreactivity, excessive mucus production, and alveolar enlargement. ${ }^{9}$ Acrolein contains a reactive carbonyl group and electrophilic carbon; thus, it is highly reactive with biological macromolecules. ${ }^{19}$ It is one of the most potent activators of respiratory sensory nerve endings, including $\mathrm{Ca}^{2+}$-permeable transient receptor potential cation channel, subfamily A, member 1 (TRPA 1$){ }^{20}$ Acrolein-activated TRPA1 signaling can lead to oxidative stress and inflammation. ${ }^{19,20}$ The present study demonstrated a change in acrolein levels in the lung tissue supernatants of non-COPD smokers and COPD patients with a verification in pathology by using immunohistochemistry that showed the expression of acrolein was increased in lung tissues of both non-COPD smokers and COPD patients.

The levels of plasm acrolein were correlated with the pack-years and with the levels of oxidants (d-ROM). However, we did not detect any correlations between smoking history and d-ROM in the COPD group in plasma study (total COPD, $\mathrm{R}=0.15$; Stage I and II, $\mathrm{R}=0.17$; and Stage III and IV, $\mathrm{R}=0.07)$. In the lung tissue study, there is no significant differences between the non-COPD smokers and COPD patients in terms of the smoking history and dROM levels. However, the lung acrolein levels were correlated with the oxidative stress in COPD group. One of the reasons might be related to the endogenous acrolein produced from neutrophil activation via myeloperoxidase and polyamine metabolism. At present, we have no available method to test the endogenous acrolein. We tried to investigate myeloperoxidase-related gene polymorphisms with plasma acrolein concentration in the present COPD group, but no positive data was obtained. We expect further study for better understanding of the mechanisms underlying the imbalance of oxidative stress and anti-oxidant biological potentiality to broaden the avenues of discovery of novel treatment strategies for COPD.

One limitation of this study was that two different groups of patients with COPD were used for the plasma and lung tissue samples. It was practically impossible to invasively obtain lung tissues from patients with severe COPD. In the study on the lung tissue, the controls were non-COPD never smokers with lung cancer of adenocarcinoma and 9/10 of them were females. Because such patients with lung cancer were almost female in Japan. The second limitation was that the total sample sizes were small and the ranges of measurements large, resulting in the statistical power not being strong enough to reduce the type II error. This study did not measure the endogenous acrolein source in plasma and lung tissue supernatants due to a lack of measurement technique in our laboratory. In addition, we did not detect any positive associations of the myeloperoxidase-related gene polymorphisms with plasma acrolein concentration in the present COPD group (data not shown). Third, the subjects in each group included both former smokers and current smokers, which might influence the acrolein levels in the measurement because of exogenous acrolein in smoke. However, at least 
in the present study, we did not detect any significant differences of acrolein concentration between the current and former smokers in the non-COPD smokers and COPD group. Nevertheless, this is the first study to investigate correlations between acrolein levels and oxidative stress or antioxidant balance in both plasma and lung tissues from patients with COPD. The increased plasma acrolein levels in COPD provided an evidence of COPD being a systematic disease. ${ }^{16,17}$ Further study is needed to explore the mechanisms of acrolein signal transduction in the oxidative stress pathway in the pathogenesis of COPD.

\section{Conclusion}

In conclusion, the acrolein levels were significantly increased in plasma and in lung tissues in patients with COPD, which were in relations with the oxidative stress in patients with COPD.

\section{Disclosure}

The authors report no conflicts of interest in this work.

\section{References}

1. Vogelmeier CF, Criner GJ, Martinez FJ, et al. Global strategy for the diagnosis, management, and prevention of chronic obstructive lung disease 2017 report. GOLD executive summary. Am J Respir Crit Care Med. 2017;195:557-582. doi:10.1164/rccm.201701-0218PP

2. Rahman I. Oxidative stress in the pathogenesis of chronic obstructive pulmonary disease. Cell Biochem Biophys. 2005;43:167. doi:10.1385/ CBB:43:1:167

3. Hanaoka M, Droma Y, Chen Y, et al. Carbocisteine protects against emphysema induced by cigarette smoke extract in rats. Chest. 2011;139:1101-1108. doi:10.1378/chest.10-0920

4. Rahman I. Antioxidant therapies in COPD. Int $J$ Chron Obstruct Pulmon Dis. 2006;1:15-29.

5. Valavanidis A, Vlachogianni T, Fiotakis K. Tobacco smoke: involvement of reactive oxygen species and stable free radicals in mechanisms of oxidative damage, carcinogenesis and synergistic effects with other respirable particles. Int J Environ Res Public Health. 2009;6:445-462. doi:10.3390/ijerph6020445

6. Eiserich JP, van der Vliet A, Handelman GJ, Halliwell B, Cross CE. Dietary antioxidants and cigarette smoke-induced biomolecular damage: a complex interaction. Am J Clin Nutr. 1995;62:1490S1500S. doi:10.1093/ajen/62.6.1490S

7. Stevens JF, Maier CS. Acrolein: sources, metabolism, and biomolecular interactions relevant to human health and disease. Mol Nutr Food Res. 2008;52:7-25. doi:10.1002/mnfr.200700412
8. Antus B, Kardos Z. Oxidative stress in COPD: molecular background and clinical monitoring. Curr Med Chem. 2015;22:627-650.

9. Bein K, Leikauf GD. Acrolein - a pulmonary hazard. Mol Nutr Food Res. 2011;55:1342-1360. doi:10.1002/mnfr.201100279

10. Global Initiative for Chronic Obstructive Lung Disease. Global Strategy for the Diagnosis, Management, and Prevention of Chronic Obstructive Pulmonary Disease. NHLBI/WHO Workshop Report [Homepage on the Internet]. Bethesda: National Heart, Lung and Blood Institute; 2001. Updated 2019]. Available from: https://goldcopd.org/gold-reports/. Accessed December 19, 2018.

11. Kubota M, Kobayashi H, Quanjer PH, Omori H, Tatsumi K, Kanazawa M. Clinical pulmonary functions Committee of the Japanese Respiratory Society. Reference values for spirometry, including vital capacity in Japanese adults calculated with the LMS method and compared with previous values. Respir Investig. 2014;52:242-250. doi:10.1016/j.resinv.2014.03.003

12. Andreoli R, Manini P, Corradi M, Mutti A, Niessen WM. Determination of patterns of biologically relevant aldehydes in exhaled breath condensate of healthy subjects by liquid chromatography/atmospheric chemical ionization tandem mass spectrometry. Rapid Commun Mass Spectrom. 2003;17:637-645. doi:10.1002/ rem. 960

13. Vassalle C, Boni C, Di Cecco P, Ndreu R, Zucchelli GC. Automation and validation of a fast method for the assessment of in vivo oxidative stress levels. Clin Chem Lab Med. 2006;44:1372-1375. doi:10.1515/CCLM.2006.243

14. Benzie IF, Strain JJ. The ferric reducing ability of plasma (FRAP) as a measure of "antioxidant power": the FRAP assay. Anal. Biochem. 1996;239:70-76. doi:10.1006/abio.1996.0286

15. Uchida K, Kanematsu M, Sakai K, et al. Protein-bound acrolein: potential markers for oxidative stress. Proc Natl Acad Sci U S A. 1998;95:4882-4887. doi:10.1073/pnas.95.9.4882

16. Sievi NA, Senn O, Brack T, et al. Impact of comorbidities on physical activity in COPD. Respirology. 2015;20:413-418. doi:10.1111/resp. 12456

17. Agusti A, Soriano JB. COPD as a systemic disease. COPD. 2008;5:133-138. doi:10.1080/15412550801941349

18. Carmella SG, Chen M, Zhang Y, Zhang S, Hatsukami DK, Hecht SS. Quantitation of acrolein-derived (3-hydroxypropyl) mercapturic acid in human urine by liquid chromatography-atmospheric pressure chemical ionization tandem mass pectrometry: effects of cigarette smoking. Chem Res Toxicol. 2007;20:986-990. doi:10.1021/tx700075y

19. Moretto N, Volpi G, Pastore F, Facchinetti F. Acrolein effects in pulmonary cells: relevance to chronic obstructive pulmonary disease. Ann N Y Acad Sci. 2012;1259:39-46. doi:10.1111/j.17496632.2012.06531.x

20. Deshmukh HS, Shaver C, Case LM, et al. Acrolein-activated matrix metalloproteinase 9 contributes to persistent mucin production. $\mathrm{Am} \mathrm{J}$ Respir Cell Mol Biol. 2008;38:446-454. doi:10.1165/rcmb.20060339OC 


\section{Publish your work in this journal}

The International Journal of COPD is an international, peer-reviewed journal of therapeutics and pharmacology focusing on concise rapid reporting of clinical studies and reviews in COPD. Special focus is given to the pathophysiological processes underlying the disease, intervention programs, patient focused education, and self management

protocols. This journal is indexed on PubMed Central, MedLine and CAS. The manuscript management system is completely online and includes a very quick and fair peer-review system, which is all easy to use. Visit http://www.dovepress.com/testimonials.php to read real quotes from published authors.

Submit your manuscript here: https://www.dovepress.com/international-journal-of-chronic-obstructive-pulmonary-disease-journal 